\title{
Knowledge Based Approach in Differential Diagnosis of Intracranial Cysts -A Requisite to Avoid Unwarranted Interventions
}

\author{
Verma $A^{1}$, Moiyadi $A^{2}$, Arya $S^{1}$, Jalali $\mathbf{R}^{3}$, Menon $\mathbf{H}^{4}$, Karbhase $\mathbf{G}^{\mathbf{1}}$, Sahwale $\mathbf{P}^{\mathbf{1}}$ \\ ${ }^{1}$ Departments of Radiodiagnosis, ${ }^{2}$ Neurosurgery, ${ }^{3}$ Radiation Oncology \& ${ }^{4}$ Medical Oncology, \\ Tata Memorial Hospital \& ACTREC, Tata Memorial Centre, Mumbai-400012, India
}

\begin{abstract}
Neuroglial cyst is a rare abnormality that is usually asymptomatic and an incidental detection in imaging done for an unrelated cause. An emphasis on this message is the prime aim of the present report. We came across a patient being treated for chronic myeloid leukemia having vague neurological symptoms for which Magnetic Resonance Imaging (MRI) was done. No detectable cause explaining his symptomatology was noted on imaging. A cystic lesion was however noted in right frontal lobe which was labeled as a neuroglial cyst. No treatment was mandated as per discussion in the neuro-oncology working group of our centre. Follow up was advised to confirm the impression as an attempt to achieve a histological diagnosis would have been ethically not justified. The patient reported regularly for his chemotherapy regimen to the respective unit and was asymptomatic for the lesion till last follow up at six months.
\end{abstract}

Keywords: Chronic Myeloid Leukemia, Intracranial cysts, Neuroglial Cyst

\section{Introduction}

Benign intracranial cysts are rare developmental variants, "Neuroglial cysts" being one of the entities in this group. These have been classified separately as "tumor like lesions" and are presumed to arise from embryonal cell rests anywhere in and around neuraxis. ${ }^{1,3}$ They are uncommon, representing less than $1 \%$ of intracranial cysts and has been classified separately as "tumor-like condition" in the latest WHO classification. ${ }^{2}$ Mostly asymptomatic, they pose a diagnostic dilemma for the imaging expert, as a histological diagnosis is not possible in most cases. Appropriate

Correspondence to: Dr. Ashish Verma Department of Radiodiagnosis, Tata Memorial Hospital, Dr. E. Borges' Road, Parel, Mumbai- 400012, India. E-mail:drdnv5@gmail.com awareness of the entity and specific imaging features are the key to diagnosis which is crucial in avoiding unnecessary surgical intervention. We present a case of asymptomatic neuroglial cyst in a patient of chronic myeloid leukemia (C.M.L).

\section{Case Report}

A 62-year old male presented to the neurooncology working group of our centre with a single episode of vertigo and diplopia. The patient was being treated for C.M.L and was admitted to the respective unit for his third chemotherapy regimen. The neurological examination of the patient did not show focal sensory or motor deficit. The higher mental functions were within normal limits with the patient being well oriented to time and place. Blood and cerebrospinal fluid examinations did not reveal abnormality, over and above those accountable by his hematological 


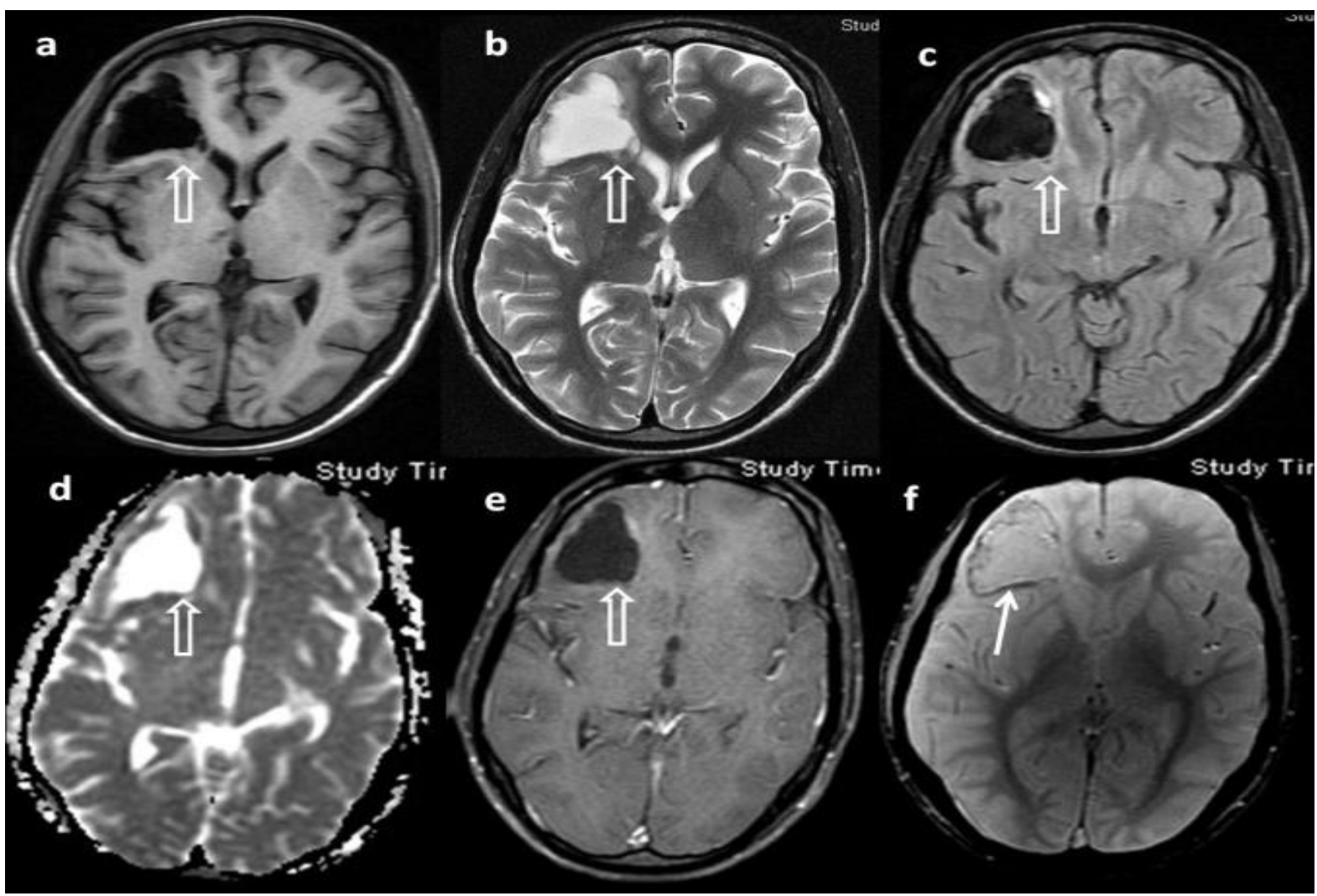

Fig 1: MRI of the patient showing a purely cystic lesion on T1W (a), T2W (b), FLAIR (c) and DWI (d) sequences. No enhancement was noted on post contrast images (e). Notable is the thin rim of calcification (straight arrows) on T2*W GRE images (f) and marginal molding along white matter tracts (hollow arrow) on all images.

malignancy. No history of recent fever, headache or head trauma at any age was available. The patient hospital records did not reveal any episode of previous cerebral hypoxia or bleed. No history of amateur or professional animal breeding was there.

MR imaging was done. Imaging was performed on a 1.5-Tesla MR system (Signa, General Electric Medical Systems, Milwaukee, WIS, USA) equipped with an actively-shielded whole body magnetic field gradient set using a quadrature birdcage receive and transmit radio frequency head coil (Fig. 1). A space occupying lesion was noted in the right basifrontal region. The lesion was well circumscribed with no secondary effect noted in the perilesional brain parenchyma. Only minimal mass effect was noted on the surrounding brain. The lesion followed CSF intensity on all sequences. No contrast enhancement was noted on administration of intravenous contrast media. The lesion was geometric in shape with marginal molding noted along the white matter tracts. A differential between neuroglial cyst, parasitic cyst and low grade cystic neoplasm was proposed. No areas of hyperperfusion, restricted diffusion or significant spectroscopic abnormality was noted. This led to further tapering down of the diagnosis to neuroglial cyst. Thin hypointense rim was noted along the wall of the cyst on $\mathrm{T} 2 * \mathrm{~W}$ GRE sequence, not visualized on any other sequence, this was most probably due to faint calcification.

The patient recalled of having a computed tomographic scan three years back following a minor road traffic accident (during which however he did not sustain any head injury). A review of previous imaging revealed a lesion similar in morphology and location with no interval change. Faint rim calcification suspected on MRI was confirmed on CT scan. In any case, this lesion could not explain patient's symptoms and it was agreed upon to observe the patient 
with supportive therapy and treatment of his primary pathology. No recurrence of neurological symptoms or any other features suggesting intracranial pathology was noted till the last documented follow up, at six months.

\section{Discussion}

Neuroglial cysts, also known as Glioependymal cysts, originate from ectopic ependymal rests at any place within or surrounding the cranio-spinal axis, like orbit, intraspinal, perispinal, sacral and pericranial. $^{3-8}$ Most common is however intracranial intracerebral location, especially frontal lobe ${ }^{1}$ as in our patient. The inner layer of these lesions consists of ependymal lining surrounded by an outer layer of neuroglial tissue (astrocytes). Above may be followed by a connective tissue layer due to vascularization. It is because of the glial coating that the name glioependymal cyst was coined. ${ }^{1,3}$

On MR imaging, these cysts are well demarcated with no definable wall or any mural nodule. The lesion contains fluid matching to cerebrospinal fluid (CSF) intensity on all sequences. Perilesional brain parenchyma (or soft tissue in case of extraaxial lesions) shows no significant changes, thin rim of resolving bleed along the walls of the only mild mass effect may be noted..$^{1-3}$ We, however report the presence of faint calcification mural calcification, not described in any of the previous reports. This was most probably due to dystrophic calcium deposition with increasing age, as the patient in our report is one of the oldest described in literature as yet.

A search of relevant contemporary clinical literature revealed most patients diagnosed of having neuroglial cysts to be asymptomatic or having unrelated symptoms. Though our patient had neurological symptoms, these could not be accounted by the imaging finding. Also these symptoms subsided without any targeted therapy and did not recur, leading to the presumption that the lesion seen on MRI was incidental. In most of these cases no surgical intervention has been advocated for the entity while decompression by minimally invasive approaches resulted in rapid refilling of the lesion by the residual mucosal lining. ${ }^{7}$ In their report of an intraspinal cyst Singh et al ${ }^{8}$, described the presence of urinary retention due to mass effect while Shenouda et $\mathrm{al}^{6}$ described a case of hemifacial spasm caused by small neuroglial cyst at the cerebellopontine angle, surgical removal of cyst can be justified in such circumstances. Few reports describing association of neuroglial cyst with arachnoid cyst ${ }^{6}$ and micro-ophthalmia ${ }^{7}$ exist in the literature, but none suggests any cause effect or genetic relationship between the entities.

The closest differential diagnostic consideration of an intra-axial neuroglial cyst is the ependymal cyst, which in most case remains strictly juxta-ventricular in location, is usually rounded and ovoid in shape and small to moderate sized. Whenever resected due to attendant complications, presence of an epithelial lining consisting of ependymal or mixed ependymal-choroidal epithelium (ciliated or non-ciliated) and a lumen filled with CSF secreted by the lining cell is the confirmatory evidence in favor of an ependymal cyst. Other possibilities include low grade neoplasia, parasitic cyst, porencephalic cyst and enlarged perivascular space all of which were ruled out on the basis of clinical history and advanced functional imaging.

The literature is overpopulated with reports, series and management strategies for arachnoids cyst, epidermoid cyst and low grade intracranial cystic neoplasm. This along with the rarity of other benign, incidental or symptomatic, intracranial cystic lesions has resulted in only few reports and short series describing the imaging features and management. We agree that our diagnosis was entirely on the basis of clinicoimaging features and follow-up with no histological evidence, but the aim of our report is to emphasize on the algorithmic 
approach based on most common anatomic locations, presentation and imaging features to narrow the differential diagnosis of intracranial cysts, as mentioned in an elegant review by Osborn AG et al. ${ }^{1}$ Such a systematic approach would be the key to evidence based management of incidental benign variants, like neuroglial cysts, and avoid unnecessary surgical intervention.

\section{References}

1. Osborn AG, Preece MT. Intracranial Cysts: Radiologic-Pathologic Correlation and Imaging Approach. Radiology 2006; 239(3):650-664.

2. Louis DN, Ohgaki H, Wiestler OD, et al. The 2007 WHO Classification of Tumors of the Central Nervous System. Acta Neuropathol2007;114:97-109.

3. Sehgal A, Singh V, Agarwal K, et al Neuroglial Cyst: A rare ocurrance in abdomen and pelvis. Ind $\mathrm{J}$ Pathol Microbiol2001;44(4):475-477.
4. Shenouda EF, Moss TH, Coakham HB. Cryptic cerebellopontine angle neuroglial cyst presenting with hemifacial spasm. Acta Neurochirurgica 2005;147(7):787789.

5. Sehgal A, Singh V, Agarwal K, et al. Neuroglial cyst: a rare occurence in abdomen and pelvis. Indian $\mathbf{J}$ Pathol Microbiol 2001; 44(4):475-477.

6. Aubry K, Wassef M, Guichard JP, et al. Association of an arachnoid cyst with heterotopic neuroglial tissue in the internal auditory canal. Ann Otolaryngol Chir Cervicofac 2009;126(3):133-137.

7. Garci ML, Castro E, Foster JA, et al. Colobomatous microphthalmia and orbital neuroglial cyst: Case report. Ophthal Genetics 2002;23(1):37-42.

8. Singh $\mathrm{N}$, Cowie $\mathrm{R}$, Pal $\mathrm{P}$, et al. Neuroglial cyst of the sacral canal. Case report. J Neurosurg Spine 2011; 14(1):8892. 